\title{
Adaptive FTC based on Control Allocation and Fault Accommodation for Satellite Reaction Wheels
}

Baldi, P.; Blanke, Mogens; Castaldi, P.; Mimmo, N.; Simani, S.

Published in:

3rd Conference on Control and Fault-Tolerant Systems (SysTol 2016)

Link to article, DOI:

10.1109/SYSTOL.2016.7739826

Publication date:

2016

Document Version

Peer reviewed version

Link back to DTU Orbit

Citation (APA):

Baldi, P., Blanke, M., Castaldi, P., Mimmo, N., \& Simani, S. (2016). Adaptive FTC based on Control Allocation and Fault Accommodation for Satellite Reaction Wheels. In 3rd Conference on Control and Fault-Tolerant Systems (SysTol 2016) IEEE. https://doi.org/10.1109/SYSTOL.2016.7739826

\section{General rights}

Copyright and moral rights for the publications made accessible in the public portal are retained by the authors and/or other copyright owners and it is a condition of accessing publications that users recognise and abide by the legal requirements associated with these rights.

- Users may download and print one copy of any publication from the public portal for the purpose of private study or research.

- You may not further distribute the material or use it for any profit-making activity or commercial gain

- You may freely distribute the URL identifying the publication in the public portal 


\title{
Adaptive FTC based on Control Allocation and Fault Accommodation for Satellite Reaction Wheels
}

\author{
P. Baldi ${ }^{1}$, M. Blanke ${ }^{2}$, P. Castaldi ${ }^{1}$, N. Mimmo ${ }^{1}$, and S. Simani ${ }^{3}$
}

\begin{abstract}
This paper proposes an active fault tolerant control scheme to cope with faults or failures affecting the flywheel spin rate sensors or satellite reaction wheel motors. The active fault tolerant control system consists of a fault detection and diagnosis module along with a control allocation and fault accommodation module directly exploiting the on-line fault estimates. The use of the nonlinear geometric approach and radial basis function neural networks allows to obtain a precise fault isolation, independently from the knowledge of aerodynamic disturbance parameters, and to design generalised estimation filters, which do not need a priori information about the internal model of the signal to be estimated. The adaptive control allocation and sensor fault accommodation can handle both temporal faults and failures. Simulation results illustrate the convincing fault correction and attitude control performances of the proposed system.
\end{abstract}

\section{INTRODUCTION}

The increasing operational requirements for on-board autonomy in satellite control systems require structural methods that support the design of complete and reliable Fault Detection and Diagnosis (FDD) and Fault Tolerant Control (FTC) systems. Significant research in FDD and FTC has been done in last decades [1], [2], [3] and many model-based methods have been proposed [4], [5]. Several fault tolerant control methods and strategies have been proposed by university and industry for aerospace applications [6], [7], [8], [9], [10]. This paper uses a detailed nonlinear satellite model to design an Active Fault Tolerant Control System (AFTCS) to cope with faults or failures affecting the reaction wheels and flywheel spin rate sensors. The procedure for actuator and sensor fault modelling presented in [11] is exploited to define a nonlinear model affine in all the actuator and sensor fault inputs and suitable for use of the NonLinear Geometric Approach (NLGA) presented in [12]. The scalar residual filters composing the model-based FDD system are designed via the NLGA to achieve fault decoupling and obtain diagnostic signals that are independent of aerodynamic disturbance parameters, and are without detection and isolation errors due to parameter uncertainties. The fault detection and isolation is achieved through a residual cross-check and a proper decision logic, assuming a single fault occurring at any time.

${ }^{1}$ P. Baldi, P. Castaldi and N. Mimmo are with the Dipartimento di Ingegneria dell'Energia Elettrica e dell'Informazione Guglielmo Marconi, Università di Bologna. 47100 Forlì (FC), Italy \{pietro.baldi2, paolo.castaldi, nicola.mimmo2\}@unibo.it

${ }^{2} \mathrm{M}$. Blanke is with the Department of Electrical Engineering, Technical University of Denmark, 2800 Kgs. Lyngby, Denmark mbelektro.dtu.dk

${ }^{3} \mathrm{~S}$. Simani is with the Dipartimento di Ingegneria, Università di Ferrara. 44123 Ferrara (FE), Italy silvio.simanidunife. it

$\mathrm{P}$. Baldi is the corresponding author.
The adaptive filters of the model-based Fault Estimation (FE) module are designed via Radial Basis Function Neural Networks (RBF NN)s [13], [14] which are enabled once a fault has been detected and isolated. The use of RBF NNs allows to design generalised fault estimation filters without a priori information about the type of fault and able to accurately estimate a generic fault without needing to define a specific fault internal model. The suggested FDD system is a substantial improvement of the ones presented in [14], [15] by the same authors. The fault sensitivity of the diagnostic filters does not depend on the satellite attitude and the number of residuals used to detect and isolate actuator and flywheel sensor faults has been reduced in comparison to [14]. Moreover, a more realistic actuator fault description has been considered in this paper, by modelling them as lossof-efficiency faults and designing proper estimation filters. The design of the AFTCS represents a further development of the previous works [14], [15], which concern the fault diagnosis. The AFTCS exploits active and adaptive reconfiguration schemes of the control actions and sensor measurements, directly making use of on-line estimates of the fault signals. Attitude control performances are maintained by exploiting redundant actuators through a Control Allocation (CA) scheme [16], [17]. Sensor signal availability is maintained by a direct sensor Fault Accommodation (FA) scheme [18]. This allows to achieve highly accurate performances for attitude control and sensor measurement. The control effort is redistributed to fault-free actuators through a reconfiguration block in the closed loop feedback system forming a fault hiding approach [19], [2]. In this way, it is possible to keep the nominal controller in the reconfigured closed-loop system, whilst the closed-loop response of the satellite remains the same, without needing to redesign the entire controller to counter each fault or failure scenario.

The system performances have been evaluated through application to a detailed nonlinear satellite simulator that includes exogenous aerodynamic and gravitational disturbance torques in addition to the nonlinear dynamics [20]. As the gravitational disturbances are almost perfectly known, the FDD robustness is achieved through an explicit disturbance decoupling based on the NLGA, applied to the aerodynamic force term, which represents the main source of uncertainty. Simulation results are shown for cases of both faults and failures affecting the reaction wheel control torques and the flywheel spin rate measurements. Finally, the ability of the scheme is assessed, regarding dealing with faults of different types, providing precise detection, isolation and estimates of faults and maintaining attitude control performance. 


\section{SPACECRAFT AND ACTUATOR MODELS}

The satellite is considered a rigid body, whose attitude is represented by using quaternion notation. The dynamic and kinematic equations of the satellite model are

$$
\begin{gathered}
\dot{\omega}=-\mathbf{I}_{s}^{-1} \mathbf{S}(\omega)\left(\mathbf{I}_{s} \omega+\mathbf{B}_{r w} \mathbf{h}_{r w}\right)+\mathbf{I}_{s}^{-1}\left(\mathbf{B}_{r w} \mathbf{M}+\mathbf{M}_{g g}+\mathbf{M}_{a e r o}\right) \\
\dot{\mathbf{q}}=\frac{1}{2} \Omega \mathbf{q}
\end{gathered}
$$

with the skew-symmetric matrices

$\mathbf{S}(\omega)=\left[\begin{array}{ccc}0 & -\omega_{3} & \omega_{2} \\ \omega_{3} & 0 & -\omega_{1} \\ -\omega_{2} & \omega_{1} & 0\end{array}\right], \Omega(\omega)=\left[\begin{array}{cccc}0 & \omega_{3} & -\omega_{2} & \omega_{1} \\ -\omega_{3} & 0 & \omega_{1} & \omega_{2} \\ \omega_{2} & -\omega_{1} & 0 & \omega_{3} \\ -\omega_{1} & -\omega_{2} & -\omega_{3} & 0\end{array}\right]$

where $\omega=\left[\omega_{1}, \omega_{2}, \omega_{3}\right]^{T}$ is the vector of the roll, pitch and yaw body rates, $\mathbf{q}=\left[q_{1}, q_{2}, q_{3}, q_{4}\right]^{T}$ is the quaternion vector and $\mathbf{h}_{r w}=\left[h_{r w_{1}}, h_{r w_{2}}, h_{r w_{3}}, h_{r w_{4}}\right]^{T}$ is the vector of the flywheel angular momenta [20]. The principal inertia bodyfixed frame is considered, with $I_{x x}, I_{y y}$, and $I_{z z}$ on the main diagonal of the satellite inertia matrix $\mathbf{I}_{s}$.

Equation (1) explicitly includes the models of the gravitational and aerodynamic disturbance torques $\mathbf{M}_{g g}$ and $\mathbf{M}_{\text {aero }}$ :

$$
\begin{aligned}
\mathbf{M}_{g g} & =\frac{3 \mu}{R^{3}}\left(\hat{v}_{\text {nadir }} \times I_{s} \hat{v}_{\text {nadir }}\right) \\
\mathbf{M}_{\text {aero }} & =\frac{1}{2} \rho S_{p} V^{2} C_{D}\left(\hat{v}_{V} \times \mathbf{r}_{c p}\right)
\end{aligned}
$$

where the parameters $\mu$ and $R$ represent the gravitational constant and the orbit radius respectively, $\hat{v}_{\text {nadir }}$ is the unit vector towards nadir expressed in satellite-body coordinates, $\rho$ is the atmospheric density, $V$ is the relative velocity of the satellite, $S_{p}$ is the reference area affected by the aerodynamic flux, and $C_{D}$ is the drag coefficient. $\mathbf{r}_{c p}=\left[r_{x_{c p}}, r_{y_{c p}}, r_{z_{c p}}\right]^{T}$ is the vector joining the centre of mass and the aerodynamic centre of pressure and $\hat{v}_{V}$ is the unit velocity vector expressed in satellite-body coordinates. It is worth noting that, mainly due to the presence of the unknown terms $\rho$ and $C_{D}$ in (5), the input $\mathbf{M}_{\text {aero }}$ in (1) represents the main source of uncertainty. These torques typically represent the most important external disturbances affecting Low Earth Orbit (LEO) satellites [20]. The design of the FDD system exploits an explicit decoupling only of the aerodynamic torque since the gravitational disturbance has a model which is almost perfectly known, and thus it does not need to be decoupled. The Attitude Control System (ACS) consists of a fixed array of four reaction wheels in a tetrahedral configuration defined by the direction vector matrix $\mathbf{B}_{r w}$ :

$$
\mathbf{B}_{r w}=\left[\begin{array}{cccc}
1 / \sqrt{3} & 1 / \sqrt{3} & -1 / \sqrt{3} & -1 / \sqrt{3} \\
\sqrt{2 / 3} & -\sqrt{2 / 3} & 0 & 0 \\
0 & 0 & -\sqrt{2 / 3} & \sqrt{2 / 3}
\end{array}\right]
$$

The elements of the input vector $\mathbf{M}=\left[M_{1}, M_{2}, M_{3}, M_{4}\right]^{T}$ correspond to the reaction wheel attitude control torques. The dynamic equations of the reaction wheel models are

$$
\dot{\omega}_{r w}=J_{r w}{ }^{-1} \dot{\mathbf{h}}_{r w}=-J_{r w}{ }^{-1}\left(\mathbf{M}+b \omega_{r w}+c \operatorname{sgn}\left(\omega_{r w}\right)\right)
$$

where $J_{r w}$ denotes flywheel inertia, $\mathbf{h}_{r w}=J_{r w} \omega_{r w}$ the vector of flywheel angular momenta, $\omega_{r w}=$ $\left[\omega_{r w_{1}}, \omega_{r w_{2}}, \omega_{r w_{3}}, \omega_{r w_{4}}\right]^{T}$ the vector of the flywheel spin rates, and $b, c$ are the viscous and Coulomb friction coefficients, respectively [21].

The overall system model is given by (1), (2) and (7). Thus, the overall state vector can be represented by $\mathbf{x}=\left[\omega_{1}, \omega_{2}, \omega_{3}, q_{1}, q_{2}, q_{3}, q_{4}, \omega_{r w_{1}}, \omega_{r w_{2}}, \omega_{r w_{3}}, \omega_{r w_{4}}\right]^{T}$ where the satellite body rates and quaternion are the controlled states and all the states are assumed measurable.

\section{FAULT DETECTION AND ISOLATION SYSTEM}

\section{A. Actuator and Sensor Fault Modelling}

The occurrence of faults or failures affecting the actuator motors and the flywheel spin rate sensors is considered in this paper. It is assumed that at most one fault can affect the system at any time.

Since (1) and (7) are affine in the control inputs, the i-th physical actuator fault can be modelled by the following fault input where $M_{c_{i}}$ is the commanded control input:

$$
F_{M i}=f_{M_{i}}=M_{c_{i}}-M_{i} \quad(i=1, \ldots, 4)
$$

Differently from [14], [15], a more realistic actuator fault description has been considered and the actuator motor faults have been actually modelled as Loss-of-Efficiency (LoE) faults with the scalar LoE factor $0 \leq k_{f_{i}} \leq=1$ :

$$
F_{M i}=f_{M_{i}}=k_{f_{i}} M_{c_{i}} \quad(i=1, \ldots, 4)
$$

If $k_{f_{i}}=0$ the i-th actuator is working perfectly whereas if $k_{f_{i}}>0$, a fault is present, and if $k_{f_{i}}=1$, the actuator has failed completely (i.e. actuator failure). Hence, it results:

$$
M_{i}=M_{c_{i}}-F_{M i}=\left(1-k_{f_{i}}\right) M_{c_{i}}=w_{r w_{i}} M_{c_{i}}
$$

where the term $w_{r w_{i}}=1-k_{f_{i}}$ represents the actual efficiency of the i-th reaction wheel. The LoE factor and effectiveness level of the four reaction wheels can be arranged into diagonal matrices $\mathbf{K}_{f}=\operatorname{diag}\left(k_{f_{1}}, \ldots, k_{f_{4}}\right)$ and $\mathbf{W}_{r w}=$ $\operatorname{diag}\left(w_{r w_{1}}, \ldots, w_{r w_{4}}\right)$, respectively.

On the other hand, the most natural way to take the occurrence of flywheel spin rate sensor faults into account, would be defining the measurement faults

$$
F_{\omega_{r w_{j}}}=\omega_{r w_{j}}-\omega_{r w_{y, j}} \quad(j=1, \ldots, 4)
$$

as the differences between the real value $\omega_{r w_{j}}$ and measured value $\omega_{r w_{y, j}}$ of the $\mathrm{j}$-th flywheel spin rate. However, this modelling would lead to the appearance of fault terms in the output equations, or more in general even to models nonlinear in the sensor fault inputs.

A different modelling procedure for sensor faults was proposed in [11] and hereby applied to obtain a dynamic model exploitable for the FDD design with a structure affine in all the fault inputs as considered by the NLGA. Essentially, it consists in introducing a suitable set of $v \geq 1$ mathematical fault inputs $f_{k}(k=1, \ldots, v)$ in place of each physical sensor fault $F$, including also a fault input associated to the time derivative of the fault $F$. Whenever a physical sensor fault 
$F \neq 0$ occurs, all the associated mathematical fault inputs $f_{k}$ will become generically nonzero, although with completely different time behaviors and, in general, without a direct physical interpretation. Hence, it will be sufficient to recognise the occurrence of any (one or more) of the associated mathematical fault inputs. For a comprehensive description of this fault modelling procedure, refer to [11].

Applying this procedure to (1), (2) and (7), a generic j-th physical flywheel spin rate sensor fault $F_{\omega_{r w}}(j=1, \ldots, 4)$ can be associated to $v_{k}=5$ mathematical fault inputs $f_{\omega_{r w_{j, k}}}$ $\left(k=1, \ldots, v_{k}\right)$ and corresponding vector fields $\ell_{\omega_{r w_{j, k}}}(x)$.

Therefore, if (1), (2) and (7) are rewritten by considering the sensor outputs $\omega_{r w_{y, j}}=\omega_{r w_{j}}-F_{\omega_{r w_{j}}}$ (i.e. $y=x+F_{x}$ ) as new state variables for the system dynamics, the general structure of a nonlinear system for the NLGA, which is affine in both the disturbance (i.e. the uncertain aerodynamic force term $d=F_{\text {aero }}$ ), actuator and sensor fault inputs, is recovered:

$$
\begin{aligned}
\dot{y}= & n(y)+p(y) d_{\text {aero }}+\sum_{i=1}^{4} g_{i}(y) M_{c_{i}}+ \\
& +\sum_{i=1}^{4} \ell_{M_{i}}(y) f_{M_{i}}+\sum_{j=1}^{4} \sum_{k=1}^{v_{k}} \ell_{\omega_{r w_{j, k}}}(y) f_{\omega_{r w_{j, k}}}
\end{aligned}
$$

\section{B. Nonlinear Geometric Approach}

The NLGA was formally developed by [12], and it relies on a coordinate change in the state and output spaces providing an observable subsystem which, if it exists, is affected by the fault, but unaffected by disturbances and the other faults to be decoupled. In the new (local) coordinates, the system can be decomposed into three subsystems $\bar{x}_{1}, \bar{x}_{2}$ and $\bar{x}_{3}$, where $\bar{x}_{1}$ is the measured part of the state affected only by the fault term $f$ to be detected, whilst $\bar{x}_{2}$ and $\bar{x}_{3}$ represent the measured and not measured part of the state affected by all the faults and disturbances, respectively. For a comprehensive description of the NLGA, refer to [12], [14], [15]. Denoting $\bar{x}_{2}$ with $\bar{y}_{2}$ and considering it as an independent input, the $\bar{x}_{1}$-subsystem can be defined as follows:

$$
\left\{\begin{array}{l}
\dot{\bar{x}}_{1}=n_{1}\left(\bar{x}_{1}, \bar{y}_{2}\right)+g_{1}\left(\bar{x}_{1}, \bar{y}_{2}\right) u_{c}+\ell_{1}\left(\bar{x}_{1}, \bar{y}_{2}, \bar{x}_{3}\right) f \\
\bar{y}_{1}=h\left(\bar{x}_{1}\right)
\end{array}\right.
$$

with $\ell_{1}\left(\bar{x}_{1}, \bar{y}_{2}, \bar{x}_{3}\right) \neq 0$. Starting from (13), a generic residual generator in filter form is modelled as follows:

$$
\left\{\begin{array}{l}
\dot{\xi}=n_{1}\left(\bar{y}_{1}, \bar{y}_{2}\right)+g_{1}\left(\bar{y}_{1}, \bar{y}_{2}\right) u_{c}+L\left(\bar{y}_{1}-\xi\right) \\
\varepsilon=\bar{y}_{1}-\xi
\end{array}\right.
$$

where $L>0$ is the gain of the asymptotically stable residual filter and $\varepsilon$ is the generated diagnostic signal.

\section{Design of the Fault Detection and Isolation Module}

Since the flywheel spin rate measurements are assumed to be available, it is straightforward to design four simple scalar NLGA residual filters independent of the aerodynamic disturbance and satellite attitude and exploiting information provided only by the reaction wheel sensors, directly on the basis of (7). Each of these NLGA residual filters results to be sensitive only to the couple of faults $f_{M_{i}}=F_{M_{i}}$, $f_{\omega_{r w_{j, 1}}}=F_{\omega_{r w_{j}}}(i=j)$, i.e. the actuator and flywheel spin rate sensor faults related to the same i-th reaction wheel, respectively, and the fault input $f_{\omega_{r w_{j}, 5}}=\dot{F}_{\omega_{r w_{j}}}$, i.e. the time derivative of the physical sensor fault. These four filters allow the isolation of the reaction wheel subsystem affected by a possible actuator or flywheel spin rate sensor fault, but not the exact and complete fault isolation. An additional NLGA residual filter is designed to allow the classification of a detected fault. On the basis of (1) and (7), the fifth filter is decoupled from the aerodynamic disturbance, i.e. not subject to detection errors due to aerodynamic parameter uncertainties. It exploits all the sensor measurements and it is insensitive to any possible actuator fault and sensitive to all the physical flywheel spin rate sensor faults through the associated mathematical fault inputs. The use of only these five residual filters characterised by fault sensitivities not depending on the satellite attitude represents a substantial improvement of the scheme presented in [14]. The scalar state variables $\xi$ of the five NLGA residual filters are

$$
\begin{array}{lll}
\xi_{1}= & J_{r w} \omega_{r w_{1}} / b & \xi_{2}=J_{r w} \omega_{r w_{2}} / b \\
\xi_{3}= & J_{r w} \omega_{r w_{3}} / b & \xi_{4}=J_{r w} \omega_{r w_{4}} / b \\
\xi_{5}= & r_{x_{c p}}\left(I_{x x} \omega_{1}+\mathbf{B}_{1} \mathbf{h}_{r w}\right)+r_{y_{c p}}\left(I_{y y} \omega_{2}+\mathbf{B}_{2} \mathbf{h}_{r w}\right)+ \\
& +r_{z c p}\left(I_{z z} \omega_{3}+\mathbf{B}_{3} \mathbf{h}_{r w}\right) &
\end{array}
$$

where $\mathbf{B}_{1}, \mathbf{B}_{2}$ and $\mathbf{B}_{3}$ are the rows of the matrix $\mathbf{B}_{r w}$.

\section{Residual Cross-check Scheme}

Assuming a single fault at any time, possible faults affecting the actuated torques or the flywheel spin rate measurements can be detected and isolated by cross-checking the five residuals $\varepsilon_{1}, \ldots, \varepsilon_{5}$ of the NLGA filters exploiting the variables (15) described in Section III-C, as follows:

1) Firstly, the first four residuals are analysed, which are sensitive only to possible actuator and sensor faults affecting a specific reaction wheel. Thus, the faulty actuator subsystem can be detected and isolated.

2) Then, the fifth residual, which is sensitive only to sensor faults and insensitive to actuator faults, is checked to precisely determine which fault has occurred.

Finally, due to the presence of measurement noise, residual thresholds have to be properly selected to minimise the false alarm and missed detection probabilities.

\section{FAULT DIAGNOSIS SYSTEM}

\section{A. Radial Basis Function Neural Network}

For a sufficiently large number $N$ of hidden-layers neurons and if the system state $x$ takes on values in a compact set $X \subset \mathscr{R}^{\ell_{n}}$, a weight matrix $W$ can be found such that a generic continuous function $f(x)$ can be approximated by RBFs, with a guaranteed finite model error $e^{*}$ [13], [14], [15]:

$$
f(x)=W \varphi(x)+e(x)=\sum_{k=1}^{N} w_{k} \varphi_{k}(x)+e(x)
$$

where $W$ is an optimal weight matrix, $\varphi_{k}$ is $k$-th RBF and $e(x)$ is the model approximation error satisfying $|e(x)| \leq e^{*}$. In this paper, the RBFs are modelled as Gaussian functions:

$$
\varphi_{k}(\hat{x})=\exp \left(-\left|\hat{x}-\mu_{k}\right|^{2} / \sigma_{k}^{2}\right)
$$

where the $k$-th RBF has center $\mu_{k}$ and width $\sigma_{k}$. 


\section{B. Estimation of Actuator and Sensor Faults}

Considering the occurrence of decreases in the actuator efficiency levels, the model (14) of each of the first four NLGA residual filters illustrated in Section III-C is modified. The purpose is to design four independent scalar RBF NN adaptive filters for the direct estimation of the LoE factor $k_{f_{i}}$ of the actuator fault $F_{M_{i}}$. This is done as follows:

$\left\{\dot{\xi}=n_{1}\left(\bar{y}_{1}, \bar{y}_{2}\right)+g_{1}\left(\bar{y}_{1}, \bar{y}_{2}\right) M_{c}+\ell_{1}\left(\bar{y}_{1}, \bar{y}_{2}\right) f+L\left(\bar{y}_{1}-\xi\right)\right.$ $\left\{\varepsilon=\bar{y}_{1}-\xi\right.$

where $\hat{f}=\hat{F}_{M_{i}}=\hat{k}_{f_{i}} M_{c_{i}}$ and the LoE factor $k_{f_{i}}$ of the fault function $f=F_{M i}(i=1, \ldots, 4)$ is estimated by the following RBF NN and adaptive law for the weight matrix $\hat{W}$ :

$$
\hat{k}_{f_{i}}=\hat{W} \varphi\left(M_{c}\right) \quad \dot{\hat{W}}=\operatorname{sgn}\left(M_{c}\right) \eta D \varepsilon \varphi^{T}\left(M_{c}\right)
$$

where $\eta>0$ is the learning ratio and $D$ is a proper constant matrix such that (18) is asymptotically stable. Considering the occurrence of flywheel spin rate sensor faults or failures, the model (14) of each of the first four NLGA residual filters illustrated in Section III-C is modified in the same way as (18), where the combined mathematical sensor fault function $f=b F_{\omega_{r w_{j}}} / J_{r w}+\dot{F}_{\omega_{r w_{j}}}(j=1, \ldots, 4)$ is estimated by the following RBF NN and weight matrix adaptive law:

$$
\hat{f}=\hat{W} \varphi(\xi) \quad \dot{\hat{W}}=\eta D \varepsilon \varphi^{T}(\xi)
$$

Then, the combined fault estimate $\hat{f}=b \hat{F}_{\omega_{r w_{j}}} / J_{r w}+\hat{\dot{F}}_{\omega_{r w_{j}}}$ is filtered by means of a first order filter with transfer function $1 /\left(s+b / J_{r w}\right)$ to derive the estimate of the actual physical sensor fault $F_{\omega_{r w_{j}}}$. These RBF adaptive filters represent an improvement of those presented in [14], [15].

\section{ACTIVE FAULT-TOLERANT CONTROL SYSTEM}

\section{A. Design of the Control Allocation Module}

The virtual control input vector $v$ can be defined as the total control effort of the actuators with respect to the satellite body axes:

$$
v=\mathbf{B}_{r w} \mathbf{u}=\mathbf{B}_{r w} \mathbf{M}
$$

A standard LQG Proportional-Integral (PI) feedback controller has been designed on the basis of the linearised satellite model to provide the nominal virtual control input vector $v_{\mathbf{c}}$ required to perform the desired attitude manoeuvre in the fault-free condition. Direct manipulation of (21) gives

$$
\mathbf{M}=\mathbf{B}_{r w}^{\dagger} \boldsymbol{v}
$$

where $\mathbf{B}_{r w}^{\dagger}$ is the right pseudo-inverse of $\mathbf{B}_{r w}$, so that $\mathbf{B}_{r w} \mathbf{B}_{r w}^{\dagger}=I_{3}$. The choice of $\mathbf{B}_{r w}^{\dagger}$ is not unique. One choice is obtained from the following minimisation problem [16]:

$$
\min _{M}\left(\mathbf{M}^{\mathbf{T}} \mathbf{W}_{r w}{ }^{-1} \mathbf{M}\right) \text { subject to } \mathbf{B}_{r w} \mathbf{M}=v
$$

where $\mathbf{W}_{\mathbf{r w}}$ is a symmetric positive definite diagonal weighting matrix which models the efficiency levels of the actuators and minimises at each time instant the weighted sum of squares cost associated with the control vector $\mathbf{M}$. The optimal solution to (23) is $\mathbf{M}=\mathbf{B}_{r w}^{\dagger} v$ where

$$
\mathbf{B}_{r w}^{\dagger}=\mathbf{W}_{r w} \mathbf{B}_{r w}{ }^{T}\left(\mathbf{B}_{r w} \mathbf{W}_{r w} \mathbf{B}_{r w}{ }^{T}\right)^{-1}
$$

In the nominal fault-free condition $\mathbf{W}_{r w}$ is set to the identity, which gives the classical Moore-Penrose pseudoinverse.

On the other hand, once the occurrence of an actuator loss-of-efficiency has been correctly isolated and estimated by the FDD system, the AFTCS properly reallocates the control effort on the redundant fault-free actuators by adaptively computing at each time instant the matrix $\hat{\mathbf{W}}_{r w}=$ $\operatorname{diag}\left(\hat{w}_{r w_{1}}, \ldots, \hat{w}_{r w_{4}}\right)=I_{4}-\operatorname{diag}\left(\hat{k}_{f_{1}}, \ldots, \hat{k}_{f_{4}}\right)$ such that the associated control component $M_{c_{i}}$ is properly weighted to minimise the use of the faulty actuator. The control signals from the desired nominal virtual control input vector $v_{c}$ are redistributed to the fault-free actuators without needing to redesign the nominal controller (i.e. implementing a fault hiding approach) [19], [2], [16]. In the event of total failure of the $\mathrm{i}$-th reaction wheel, $M_{c_{i}}(i=1, \ldots, 4)$ is totally rerouted to the other reaction wheels assuming a sufficient actuator redundancy. Hence, the satellite is able to achieve the desired pointing condition, while reducing the performance degradation due to the fault situation and avoiding mission abortion. Thanks to the assumed configuration and redundancy of the actuators, different solutions for control reallocation are possible to compensate the occurrence of single actuator faults, achieve the required total control effort and obtain the desired attitude control performances. Using (22) and (24), the allocated control inputs commanded to each actuator are

$$
\mathbf{M}_{c}=\mathbf{W}_{r w} \mathbf{B}_{r w}^{T}\left(\mathbf{B}_{r w} \mathbf{W}_{r w}{ }^{2} \mathbf{B}_{r w}{ }^{T}\right)^{-1} v_{c}
$$

and, due to the occurred decrease in actuator efficiency, the actual control torques provided by the actuator motors are

$$
\mathbf{M}=\mathbf{W}_{r w} \mathbf{M}_{c}
$$

with the resulting total control effort defined as

$$
v=\mathbf{B}_{r w} \mathbf{M}
$$

\section{B. Design of the Sensor Fault Accommodation Module}

Once the detection and isolation of the occurred flywheel spin rate sensor fault or failure $F_{\omega_{r w_{j}}}$ has been achieved, the obtained estimate $\hat{F}_{\omega_{r w_{j}}}$ is directly injected into the feedback control loop to compensate the effect of the sensor fault or failure [18]. Starting from (11), the accommodated sensor measurements $\omega_{r w_{a c c, j}}(\mathrm{j}=1, \ldots, 4)$ are given by

$$
\omega_{r w_{a c c, j}}=\omega_{r w_{y, j}}+\hat{F}_{\omega_{r w_{j}}}=\omega_{r w_{j}}-F_{\omega_{r w_{j}}}+\hat{F}_{\omega_{r w_{j}}}
$$

\section{SIMULATION RESULTS}

It is supposed that $\mathbf{r}_{c p}=[0.10,0.15,-0.35] \mathrm{m}, C_{D}=2.2$, $I_{x x}=330 \mathrm{~kg} \cdot \mathrm{m}^{2}, I_{y y}=280 \mathrm{~kg} \cdot \mathrm{m}^{2}, I_{z z}=60 \mathrm{~kg} \cdot \mathrm{m}^{2}, J_{r w}=$ $0.05 \mathrm{~kg} \cdot \mathrm{m}^{2}$ and $\omega_{r w_{j}}(t=0)=-300 \mathrm{rpm}$. A circular equatorial low Earth orbit at an altitude of $350 \mathrm{~km}$ with $R=$ $6728.140 \mathrm{~km}, \rho=\rho_{\max }=6 \cdot 10^{-11} \mathrm{~kg} / \mathrm{m}^{3}, V=8187.63 \mathrm{~m} / \mathrm{s}$ and $\mu=39.86004418 \cdot 10^{13} \mathrm{~m}^{3} / \mathrm{s}^{2}$ is considered.

Sensor noises are modelled by Gaussian processes with zero mean and standard deviations equal to $3 \operatorname{arcsec}, 3 \operatorname{arcsec} / \mathrm{s}$ and $1 \mathrm{rpm}$ for the attitude expressed in Euler angles, satellite angular velocity and flywheel spin rates, respectively. An attitude change manoeuvre is considered, commencing at 
$t_{\text {man }}=5 \mathrm{~s}$ from the initial attitude expressed in Euler angles equal to $\left[\phi_{0}, \theta_{0}, \psi_{0},\right]^{T}=[-15,35,25]^{T}$ deg to the final one $[\phi, \theta, \psi,]^{T}=[-10,30,25]^{T} \mathrm{deg}$.

Assuming a single fault at any time, three additive fault scenarios commencing at $t_{\text {fault }}=10 \mathrm{~s}$ are considered:

1) complete actuator failure: $F_{M_{2}}=k_{f_{2}} M_{c_{2}}$ with $k_{f_{2}}=1$;

2) actuator loss-of-efficiency fault: $F_{M_{2}}=k_{f_{2}} M_{c_{2}}$ with $k_{f_{2}}=0.3+0.05 \sin (2 \pi t / 60)$;

3) flywheel spin rate sensor fault: $F_{\omega_{r w_{2}}}=a_{\omega_{r w}} \omega_{r w_{2}}+b_{\omega_{r w}}$ with $a_{\omega_{r w}}=0.05, b_{\omega_{r w}}$ passing from zero at $t=10 \mathrm{~s}$ to $-0.5235 \mathrm{rad} / \mathrm{s}=-100 \mathrm{rpm}$ at $t=20 \mathrm{~s}$.

Figs. 1 and 2 show the diagnostic signals of the second and fifth residual filters described in Section III-C in the first and third fault scenarios, respectively. In the second fault scenario, a result similar to Fig. 1 is obtained. In particular, the residual $\varepsilon_{2}$ is sensitive to the couple of faults $F_{M_{2}}, F_{\omega_{r w_{2}}}$, whilst the residual $\varepsilon_{5}$ is sensitive only to $F_{\omega_{r w_{2}}}$ and decoupled from the aerodynamic disturbance. Due to the lack of space, the remaining three residuals, which do not exceed the selected thresholds, are not shown. Since each of the four residuals $\varepsilon_{i}(i=1, \ldots, 4)$ is sensitive only to the actuator and sensor faults on a specific reaction wheel subsystem, it is possible to detect and isolate the faulty reaction wheel subsystem as described in Section III-D. Then, a check on the residual $\varepsilon_{5}$ allows to precisely isolate also the type of the occurred fault since this residual is sensitive only to sensor faults and insensitive to actuator faults. As shown, $\varepsilon_{5}$ exceeds its thresholds only after the occurrence of a sensor fault. Moreover, both the residuals return to their initial conditions within the thresholds once the failed actuator has been excluded and the corresponding control input completely rerouted to the remaining working actuators, or the sensor fault has been accommodated.

Once the occurred fault or failure has been detected and isolated, the corresponding RBF NN adaptive estimation filter is activated. Fig. 3 shows the obtained accurate estimates of the actual LoE factors for the actuator failure and fault, and of the flywheel spin rate sensor fault, respectively.

Fig. 4 shows the control inputs commanded to each reaction wheel with and without control reallocation in case of actuator failure. The actual control torque provided by the failed actuator is depicted in red. After the failure, the control input of the second wheel is completely rerouted to the other working actuators and the failed actuator is effectively disabled without needing to redesign the attitude controller. Fig. 5 shows the total control effort provided by the actuators with respect to the satellite body axes, in the nominal faultfree condition and in case of actuator failure with and without control reallocation. The total control effort required to perform the desired manouvre is achieved thanks to the actuator redundancy and an accurate control reallocation.

Fig. 6 shows the behaviours of the roll, pitch and yaw angles $\phi, \theta, \psi$ obtained with and without control reallocation after the actuator failure. The attitude presents a large deviation from the desired reference values, or even an instability, if the control reallocation is not present. On the contrary, if the

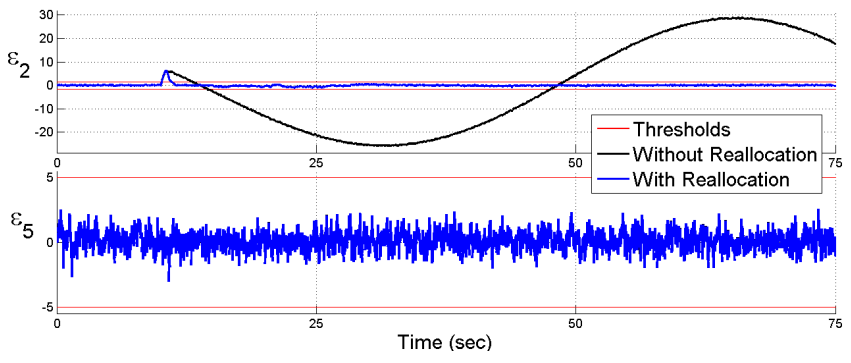

Fig. 1. Actuator failure: residual sensitive to faults on the second reaction wheel and residual sensitive only to flywheel spin rate sensor faults.

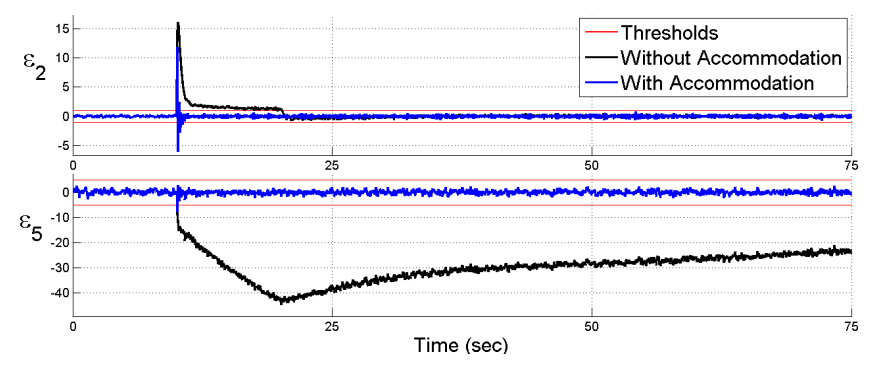

Fig. 2. Flywheel sensor fault: residual sensitive to faults on the second reaction wheel and residual sensitive only to flywheel spin rate sensor faults.

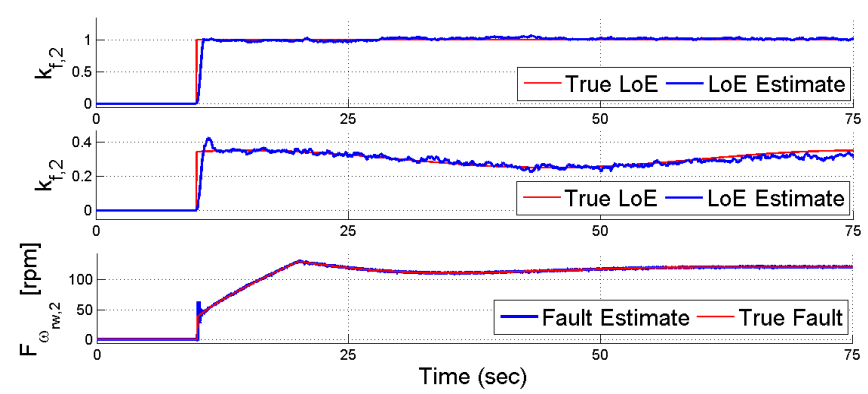

Fig. 3. Estimates of the LoE factor $k_{f_{2}}$ and physical sensor fault $F_{\omega_{r w_{2}}}$ for the three considered fault scenarios.

control inputs are properly rerouted, the desired attitude can be accurately reached as in the fault-free case.

Fig. 7 shows the behaviours of the measured $\omega_{r w_{2}}$, with and without direct fault accommodation. With the active fault accommodation, the nominal behaviour of the sensor output obtainable in the fault-free condition is accurately restored. Finally, Fig. 8 shows the results of a stability analysis performed through a series of 10 simulations with different initial attitude conditions and fault severities, i.e. different values of the actuator LoE factor $k_{f_{2}}$.

\section{CONCLUSION}

This paper presented an active fault tolerant control scheme for the attitude control system of a low Earth orbit satellite. Fault diagnosis with robust disturbance decoupling was achieved using a nonlinear geometric approach. The use of radial basis function neural networks was further shown to allow for design of generalised fault estimation filters, able to estimate a generic fault without needing prior information about the fault internal model. The control reconfiguration 
and sensor fault accommodation exploited directly the online estimates of the different fault signals. The simulation

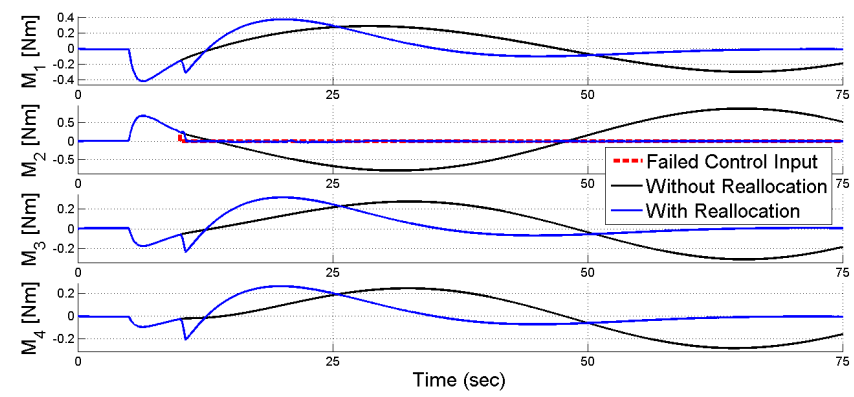

Fig. 4. Actuator failure: control inputs commanded to each reaction wheel.

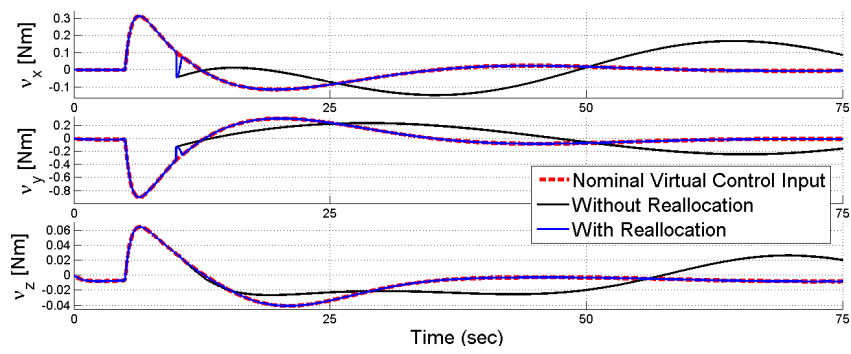

Fig. 5. Actuator failure: total control effort w.r.t. the satellite body axes.

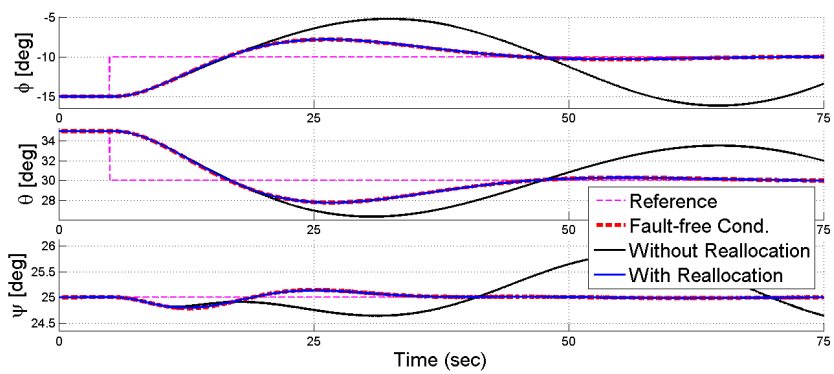

Fig. 6. Actuator failure: attitude with and without control reallocation.

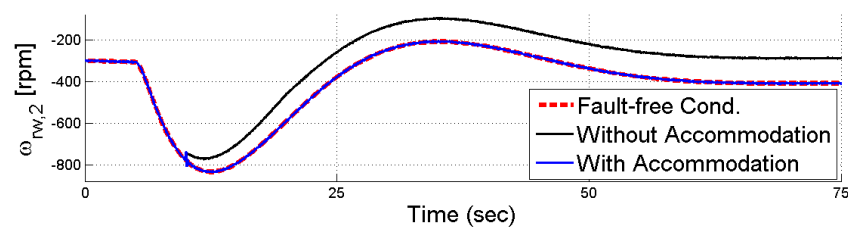

Fig. 7. Flywheel spin rate sensor fault: flywheel spin rate sensor output.

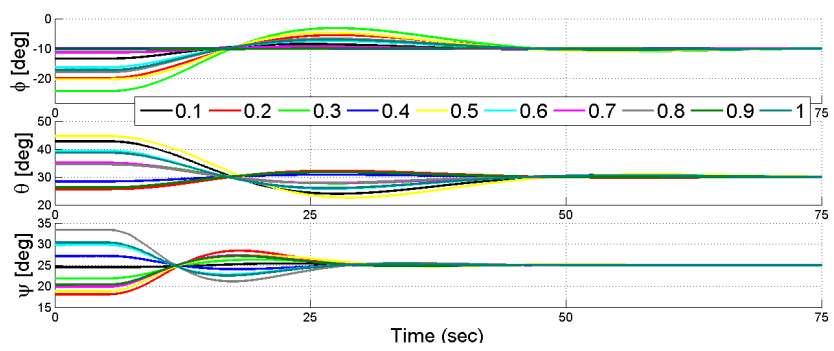

Fig. 8. Actuator faults: stability analysis of satellite attitude with control reallocation for different values of loss-of-efficiency and initial conditions. results highlighted the efficacy of the proposed scheme to achieve a precise fault detection and isolation, provide accurate fault estimates and perform a control reallocation or sensor fault accommodation to compensate the fault and failure effects. Further investigations will concern the analytical demonstration of stability of the proposed system.

\section{REFERENCES}

[1] R. Isermann, Fault Diagnosis Applications: Model-based Condition Monitoring: Actuators, Drives, Machinery, Plants, Sensors, and Faulttolerant Systems. Springer Berlin Heidelberg, 2011.

[2] M. Blanke, M. Kinnaert, J. Lunze, and M. Staroswiecki, Diagnosis and Fault-tolerant Control, 3rd Edition. 3rd Ed., Springer Berlin Heidelberg, 2016

[3] Y. Zhang, J. Jiang, "Bibliographical Review on Reconfigurable FaultTolerant and Control Systems," Annual Reviews in Control, vol. 32, no. 2, pp. 229-252, December 2008.

[4] J. Marzat, H. Piet-Lahanier, F. Damongeot, and E. Walter, "Modelbased fault diagnosis for aerospace systems: a survey," Proc. of the Institution of Mechanical Engineers, Part G: Journal of Aerospace Engineering, January 2012.

[5] S. X. Ding, Model-based Fault Diagnosis Techniques: Design Schemes, Algorithms and Tools. 2nd Ed., Springer London, 2013.

[6] T. Lorentzen, M. Blanke, and H. H. Niemann, "Structural analysis - A case study of the Rømer satellite," Proc. of 5-th IFAC Symp. SAFEPROCESS'03, pp. 187-192, June 2003.

[7] X. Olive, "FDI(R) for satellite at Thales Alenia Space: How to deal with high availability and robustness in space domain?," Proc. of SysTol'10, pp. 837-842, October 2010.

[8] D. Henry, C. Le Peuvedic, L. Strippoli, and F. Ankersen, "Robust Model-based Fault Diagnosis of Thruster Faults in Spacecraft," Proc. of 9-th IFAC Symp. SAFEPROCESS'15, vol. 48, no. 21, pp. 10781083, September 2015.

[9] R. Fonod, D. Henry, C. Charbonnel, E. Bornschlegl, D. Losa, and S. Bennani, "Robust FDI for fault-tolerant thrust allocation with application to spacecraft rendezvous," Control Engineering Practice, vol. 42, pp. 12-27, September 2015

[10] S. Yin, B. Xiao, S. Ding, and D. Zhou, "A Review on Recent Development of Spacecraft Attitude Fault Tolerant Control System," IEEE Transactions on Industrial Electronics, 2016.

[11] R. Mattone, and A. De Luca, "Nonlinear fault detection and isolation in a three-tank heating system," IEEE Trans. on Control Systems Technology, vol. 14, no. 6, pp. 1158-1166, November 2006.

[12] C. De Persis, and A. Isidori, "A Geometric Approach to Nonlinear Fault Detection and Isolation," IEEE Transactions on Automatic Control, vol. 45, no. 6, pp. 853-865, June 2001.

[13] T. Chen, and H. Chen, "Approximation capability to functions of several variables, nonlinear functionals, and operators by radial basis function neural networks," IEEE Trans. on Neural Networks, vol. 6, no. 4, pp. 904-910, July 1995.

[14] P. Baldi, M. Blanke, P. Castaldi, N. Mimmo, and S. Simani, "Combined Geometric and Neural Network Approach to Generic Fault Diagnosis in Satellite Reaction Wheels," Proc. of 9-th IFAC Symp. SAFEPROCESS'15, vol. 48, no. 21, pp. 194-199, September 2015.

[15] P. Baldi, M. Blanke, P. Castaldi, N. Mimmo, and S. Simani, "Combined Geometric and Neural Network Approach to Generic Fault Diagnosis in Satellite Actuators and Sensors," 20-th IFAC Symp. ACA 2016, Sherbrooke, Quebec, Canada, 21-25 August 2016.

[16] H. Alwi, C. Edwards, and C. P. Tan, Fault Detection and FaultTolerant Control Using Sliding Modes. Springer, 2011.

[17] T. A. Johansen, and T. I. Fossen, "Control Allocation - A Survey," Automatica, vol. 49, no. 5, pp. 1087-1103, May 2013.

[18] K. Zhang, B. Jiang, and P. Shi, Observer-Based Fault Estimation and Accommodation for Dynamic Systems. Springer Berlin Heidelberg, 2013.

[19] J. Lunze, "Control Reconfiguration After Actuator Failures: The Generalised Virtual Actuator," Proc. of 6-th IFAC Symp. SAFEPROCESS'06, vol. 6, pp. 1240-1245, August 2006.

[20] B. Wie, Space Vehicle Dynamics and Control (2nd ed.). AIAA Education Series, 2008

[21] V. Carrara, A. G. da Silva, and H. K. Kuga, "A dynamic friction model for reaction wheels," 1-st IAA Conference on Dynamic and Control of Space Systems - DyCoSS'2012, vol. 145, pp. 343-352, March 2012. 